Check for updates

Cite this: RSC Adv., 2018, 8, 12449

Received 10th January 2018

Accepted 20th March 2018

DOI: $10.1039 / \mathrm{c} 8 \mathrm{ra00260f}$

rsc.li/rsc-advances

\title{
Transcriptome analysis reveals GA induced apoptosis in HCT116 human colon cancer cells through calcium and p53 signal pathways
}

\author{
Chen Yang, (D) *abc Xiaoyu Xie, ${ }^{d}$ Hu Tang, (D) abc Xuyan Dong, ${ }^{\text {abc }}$ Xiaodong Zhang $^{d}$ \\ and Fenghong Huang*abc
}

Gallic acid (GA) is a polyphenol widely found in numerous fruits and vegetables that has been reported to exert anticancer effects, including apoptosis, against cancer cell lines. However, little is known about the induction of apoptotic effects and the underlying mechanism. We used RNA-seq to examine differentially expressed genes in human colon cancer HCT116 cells after $12 \mathrm{~h}$ and $24 \mathrm{~h}$ exposure to GA. A total of 792 and 911 genes with known functions showed significantly different expression levels in $12 \mathrm{~h}$ and $24 \mathrm{~h}$ GA-treated HCT116 cells, respectively. KEGG enrichment analysis showed that the identified genes were involved in pathways such as cholinergic synapse, circadian entrainment, calcium signal processing and transport, arachidonic acid metabolism and the p53 signal pathway. Real-time quantitative PCR was used to validate the reliability of the results obtained by RNA-seq. The results of this study indicate that GA triggers apoptosis in HCT116 cells through obstructing the growth of cells in the early phase treatment by down-regulation of calcium channels and then up-regulation of the intrinsic p53 signal pathway through activation of apoptosis caspases, finally leading to the mitochondrial apoptosis pathway.

\section{Introduction}

Colorectal cancer (CRC) is the third most common type of cancer and the third leading cause of cancer-related death in the United States. ${ }^{\mathbf{1}}$ CRC is common in both men and women and occurs when malignant tumors form on the inner wall of the large intestine. ${ }^{2}$ Epidemiological studies show that most cases $(\sim 85 \%)$ of CRC are considered to be sporadic cases rather than due to hereditary factors. ${ }^{3}$ The sporadic development of colon cancer is tightly linked to environmental factors and individual lifestyle factors, including dietary habits, alcohol consumption and physical exercise. ${ }^{4}$ The conventional treatment of colon cancer includes surgery, chemotherapy and radiofrequency ablation; however, the effectiveness of chemotherapy is hampered by the disadvantages of toxicity and drug resistance. ${ }^{5}$ Thus, the identification of drugs with lower toxicity, such as plant-derived bioactive compounds, that might play a role in the remedy or prevention of CRC is vital. ${ }^{6,7}$

\footnotetext{
${ }^{a}$ Oil Crops Research Institute, Chinese Academy of Agricultural Sciences, Wuhan 430062, P. R. China.E-mail:yangchen@caas.cn; huangfh@oilcrops.cn

${ }^{b}$ Key Laboratory of Oilseeds Processing, Ministry of Agriculture, Wuhan 430062, P. R. China

${ }^{c}$ Hubei Key Laboratory of Lipid Chemistry and Nutrition, Wuhan 430062, P. R. China ${ }^{d}$ Department of Cell Biology, College of Life Sciences, Wuhan University, Wuhan 430072, P. R. China
}

Hydroxycinnamic and hydroxybenzoic acids are common dietary phenolic acids derived from many edible plants and micronutrients that exhibit significant biological activities in the human body. ${ }^{8}$ Gallic acid (GA) is a model hydroxybenzoic acid that is widely distributed in many edible plants such as vegetables, several legumes, fruits, hickory nuts. ${ }^{\mathbf{9 1 0}}$ GA displays marked biological activities, including antioxidative, ${ }^{\mathbf{1 1}}$ antiinflammatory, ${ }^{12}$ antibacterial, ${ }^{13}$ and anti-virus activities. ${ }^{14}$ In recent years, GA has attracted much interest from scientists due to its capability of inducing apoptosis in several types of cancer cell lines, including HL-60RG, ${ }^{15}$ LL-2, ${ }^{16}$ MCF-7 (ref. 17) and SCC4 (ref. 18) cells. In particular, a previous report showed that GA could induce cell death in CoLo205 and HCT-15 colon cancer lines. ${ }^{19}$ However, the apoptotic mechanism of GA in colon cancer lines has not been fully elucidated.

Over the past decade, gene expression profiles have been applied into drug development to understand and predict the activity of chemical compounds in pre-clinical test. ${ }^{20}$ High throughput data created by large scale "omic" approaches provide a comprehensive overview and easily reveal impacts on biological pathways and processes that had not previously been found before. ${ }^{21}$ RNA sequencing (RNA-seq) is an unbiased sequencing tool that uses whole-genome transcriptome profiling to detect gene expression changes in a cell or tissue sample. ${ }^{22,23}$

In this study, the apoptosis mechanism of GA was investigated in HCT116 colon cancer cells. We used RNA-seq 
technology to evaluate the changes in the gene expression profile induced by GA treatment of HCT116 colon cancer cells to identify the regulatory pathways and modulated genes that may represent potential therapeutic targets.

\section{Experimental}

\section{Reagents and cell culture}

The human cancer cell lines HCT116, RKO and SW48 were purchased from American Type Culture Collection (ATCC, Manassas, VA, USA). The HCT116 and RKO were cultured in McCoy's 5A (Invitrogen, Carlsbad, CA, USA), SW48 was grown in DMEM medium (HyClone, Logan, UT, USA). Both medium was supplemented with $10 \%$ fetal bovine serum (HyClone, Logan, UT, USA) and 1\% penicillin-streptomycin (Invitrogen, Carlsbad, CA, USA). The cells were incubated in $5 \% \mathrm{CO}_{2}$ at $37^{\circ} \mathrm{C}$. Cells in the logarithmic growth stage were used for the experiments. GA (Sigma Chemical, St. Louis, MO, USA) was dissolved in DMSO (Sigma Chemical, St. Louis, MO, USA) at a stock concentration of $10 \mathrm{mM}$ and then diluted in water to a working concentration.

\section{Cell viability assay}

Cells were seeded into 96-well flat-bottom plates at a density of 1 $\times 10^{4}$ per well and cultured overnight, and then treated with increasing dosages of GA for $12 \mathrm{~h}, 24 \mathrm{~h}$, or $48 \mathrm{~h}$. Cell viability was assayed using the Cell Counting Kit-8 (Dojindo Laboratories, Kumamoto, Japan) according to the manufacturer's instructions. Cells were incubated with $10 \mu \mathrm{L}$ of CCK-8 solution for $1 \mathrm{~h}$ at $37{ }^{\circ} \mathrm{C}$, and the absorbance was then determined by a spectrophotometer set ELx800 (BioTek, Winooski, VT, USA) at a wavelength of $450 \mathrm{~nm}$.

\section{Flow cytometry assay}

For apoptosis analysis, cells $\left(1 \times 10^{5}\right)$ were examined using Annexin V-FITC and PI (FITC Annexin V Apoptosis Detection Kit I, BD, CA, USA) according to the manufacturer's instructions. Briefly, HCT116 cells were collected, washed with ice-cold PBS and resuspended in $100 \mu \mathrm{L}$ binding buffer. Then, $2 \mu \mathrm{L}$ of Annexin V-FITC and $5 \mu \mathrm{L}$ of PI were added to the cells. After $15 \mathrm{~min}$ at room temperature in the dark, an additional $400 \mu \mathrm{L}$ of binding buffer was added and the cells were subjected to FACS cytometry (BD Biosciences, San Jose, CA, USA). The results were analyzed with Summit version 4.3 software.

\section{RNA extraction and cDNA synthesis}

The cells were seeded on $100 \times 20 \mathrm{~mm}$ dishes at $5 \times 10^{5}$ cells per dish and incubated overnight. Cells were harvested after treatment with $50 \mu \mathrm{M}$ GA or with $0.5 \%$ DMSO (negative control) for $12 \mathrm{~h}$ or $24 \mathrm{~h}$. Total RNA was extracted using TRIzol reagent (Life Technologies, Waltham, MA, USA) and each treatment had three duplicates. RNA purity was monitored on $1 \%$ agarose gels. RNA integrity was assessed using the RNA Nano 6000 Assay Kit of the Bioanalyzer 2100 system (Agilent Technologies, CA, USA).

\section{Library preparation and transcriptome sequencing}

Total RNA sample libraries were extracted using the Illumina TruSeq Stranded mRNA LT sample preparation kit (Illumina, San Diego, CA, USA), according to the standard manufacturer's instructions. rRNA was removed from the total RNA using the rRNA removal kit (Illumina, San Diego, CA, USA). cDNA was then synthesized from RNA using Super Script II Reverse Transcriptase (Invitrogen, CA, USA); the first strand of cDNA was synthesized with hexahedron random primers and reverse transcriptase, and the second strand cDNA was synthesized using the first strand of cDNA as template. After the library was constructed, the fragmented library was enriched by PCR amplification, and then fragments of 300-400 bp were selected. PCR was then performed with universal PCR primers and an Index (X) Primer. Finally, the PCR products were purified by AMPure XP system (Beckman coulter, Kraemer Boulevard, CA, USA) and the library quality was assessed using the Agilent Bioanalyzer 2100 system.

\section{Bioinformatic analysis}

Transcriptome sequencing was performed by Shanghai Personal Biotechnology Co., Ltd. (Shanghai, China) based on the Illumina HiSeq platform using a 150 cycle paired-end sequencing strategy. FastQC was used for quality control; a quality score $Q>20$ of reads were used for downstream analysis. The reference genome sequence was downloaded from the Ensembl Genome Browser (http://www.ensembl.org/ index.html) as the mapping reference. The reads were mapped against the reference using TopHat v2.0.1028,29 and Bowtie v2.1.030 after quality control.

Bioinformatics analysis. HTSeq v0.6.1 was used to count the numbers of reads mapped to each gene and gene expression levels were presented as RPKM (reads per kilobase of exon model per million mapped reads). We used the DESeq (version 1.18.0) to detect DEGs with the gene symbol annotation (FDR < 0.05 , fold change $>2$ ). In addition, principle component analysis (PCA) was carried out on the significantly expressed genes among all different groups. The Gene Ontology (GO) annotations curated by the European Bioinformatics Institute were imported in the CLC Genomics Workbench v.7.0 and annotations of the DEGs were processed. Enrichment analysis of DEGs was conducted using the Kyoto Encyclopedia of Genes and Genomes (KEGG) pathway enrichment analysis.

\section{Real-time quantitative PCR (qPCR)}

Reverse transcription was performed using the TaKaRa PrimeScript $^{\text {TM }}$ RT-PCR Kit (TaKaRa, Tokyo, Japan) and qRT-PCR was performed with TransStart Top Green qPCR SuperMix (TRANSGEN, Beijing, China) on the CFX384 Touch ${ }^{\mathrm{TM}}$ (Bio-Rad, Hercules, CA, USA) following the manufacturer's protocol. The following thermal profile was used: an initial 30 second denaturation step at $95{ }^{\circ} \mathrm{C}$, followed by 40 cycles at $95{ }^{\circ} \mathrm{C}$ for 15 second and at $55{ }^{\circ} \mathrm{C}$ for 15 second and finally at $72{ }^{\circ} \mathrm{C}$ for 20 second. The data were analyzed using 7500 software v.2.0.1 (Applied Biosystems, Foster City, CA, USA) for $C_{\mathrm{t}}$ determination. 
Gene expression levels were calculated using the comparative $\Delta \Delta C_{\mathrm{t}}$ method and glyceraldehyde-3-phosphate dehydrogenase (GAPDH) mRNA was used as an internal control (primer sequences $5^{\prime}-3^{\prime}$ are CCCCACCACACTGAATCTCC and GTACATGACAAGGTGCGGCT). The results are provided as the mean and standard deviation of three technical replicates.

\section{Results}

\section{Effect of GA on cell proliferation}

The effects of GA on colon carcinoma cell proliferation and growth were evaluated by CCK8 assay. As shown in Fig. 1a, GA treatment for $24 \mathrm{~h}$ remarkably decreased cell viability in HCT116 and SW48 cells in a dose-dependent manner. The susceptibility of HCT116 cells to GA was higher than that of SW48 and RKO cells. The IC $_{50}$ value of GA treatment for HCT116 cells and SW48 cells were $54.8 \mu \mathrm{M}$ and $41.4 \mu \mathrm{M}$, respectively, at $24 \mathrm{~h}$. We also found that GA had an inhibitory effect on HCT116 cells in a time-dependent manner (Fig. 1b). The viability of HCT116 cells sharply dropped at $12 \mathrm{~h}$ to $60.5 \%$ and reached $34.8 \%$ at $24 \mathrm{~h}$ upon treatment with $50 \mu \mathrm{M}$ GA. However, there were no significant differences between cell viability at $12 \mathrm{~h}$ and $24 \mathrm{~h}$ at lower GA concentrations $(12.5 \mu \mathrm{M}$ and $25 \mu \mathrm{M})$ examined.

\section{Effect of GA on apoptosis in HCT116 cells}

To further explore the mechanisms underlying the inhibitory effects of GA, cell apoptosis was assessed using Annexin V-PI dual staining and flow cytometric analysis (Fig. 2). The percentages of later apoptotic cells (in the upper right quadrant) were significantly increased by treatment with GA at $25 \mu \mathrm{M}(12.3 \pm 3.2 \%), 50 \mu \mathrm{M}$ $(62.2 \pm 4.5 \%)$, and $100 \mu \mathrm{M}(92.8 \% \pm 7.7 \%)$ for $24 \mathrm{~h}$ in comparison with the control $(7.2 \pm 0.5 \%, P<0.05)$. The early apoptotic cell proportions (in the lower right quadrant) in response to $25 \mu \mathrm{M}, 50$ $\mu \mathrm{M}$ and $100 \mu \mathrm{M}$ GA were $6.3 \pm 0.4 \%, 6.2 \pm 0.6 \%$ and $1.6 \pm 0.5 \%$, respectively. These results suggested that GA could partially inhibit the proliferation of HCT116 cells by inducing apoptosis.

\section{Effect of GA on gene expression patterns}

To determine the expression patterns of mRNAs during GA treatment, heat maps were constructed to profile the overall transcriptome differences at different time points. More than $67 \%$ of genes were expressed (RPKM $>1$ ) in each sequenced sample. Heat map representation of differently expressed transcripts revealed a similarity in the transcriptomes within the three triplicate runs of untreated group (C1, C2, C3), $12 \mathrm{~h}$ treatment group (GA12_1, GA12_2, GA12_3) and $24 \mathrm{~h}$ treatment group (GA24_1, GA24_2, GA24_3), respectively (Fig. 3a).
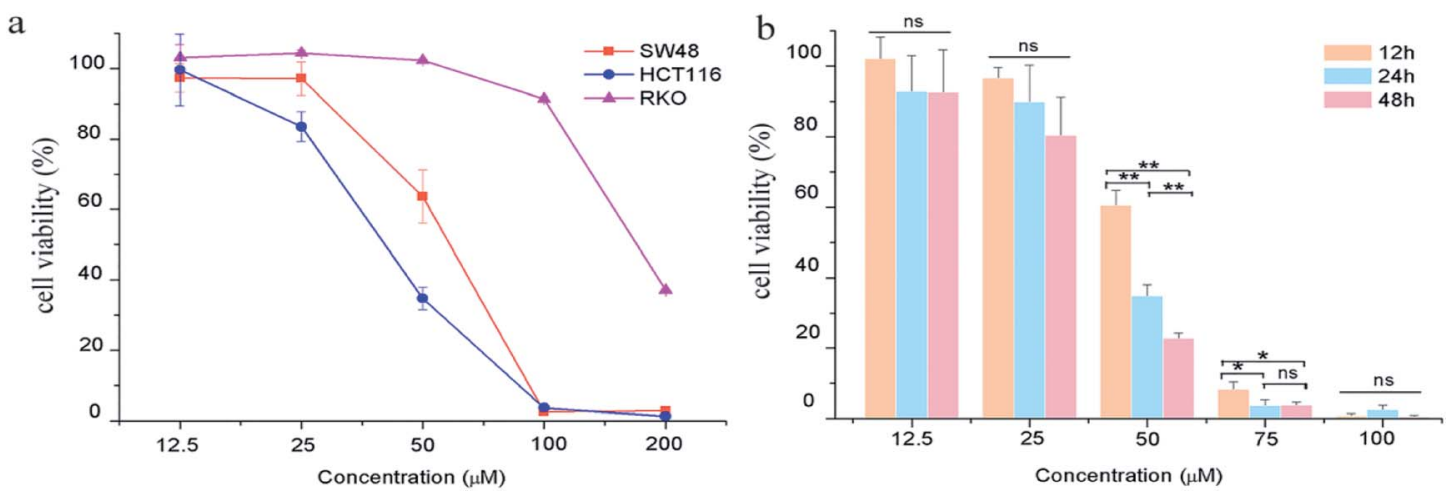

Fig. 1 The effects of GA on colon carcinoma cell proliferation. (a) The cell viability of HCT116, RKO and SW48 cells treated with GA for $24 \mathrm{~h}$. (b) The cell viability of HCT116 cells treated with GA for $12 \mathrm{~h}, 24 \mathrm{~h}$ and $48 \mathrm{~h}$. Statistically significant differences $p<0.05$ and $p<0.01$ between different treated cells are denoted by * and ** respectively, "ns" means there was no significant difference between treated and untreated cell.
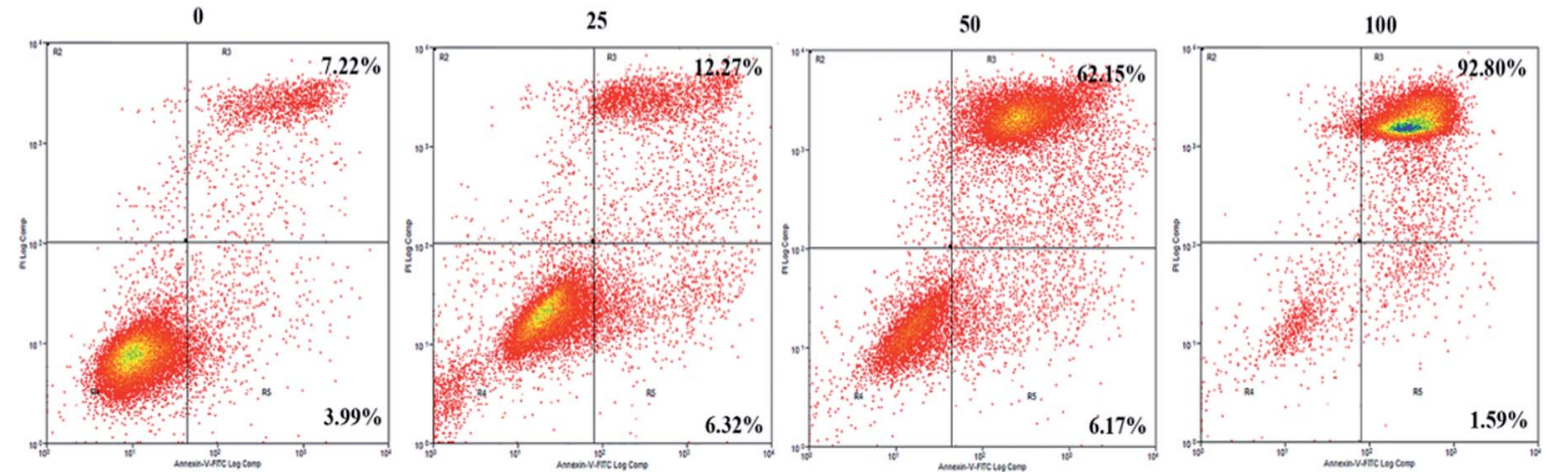

Fig. 2 The effects of GA on apoptosis of HCT116 cells. Cell incubation for $24 \mathrm{~h}$ with $0,25,50$ and $100 \mu \mathrm{M}$ of GA was followed by analysis using flow cytometry. 
a

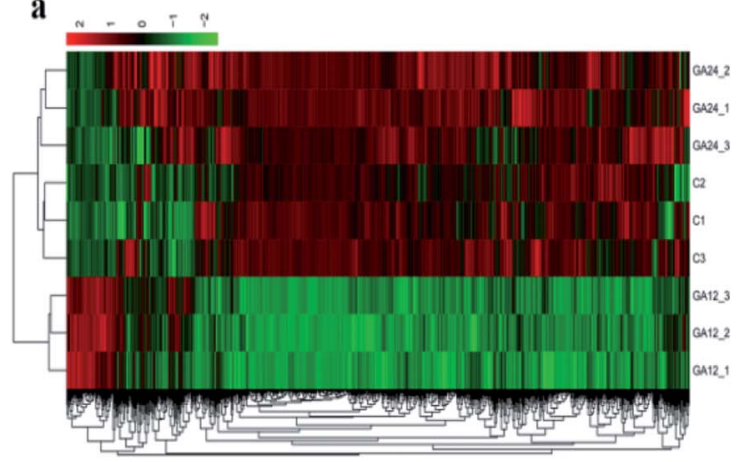

b

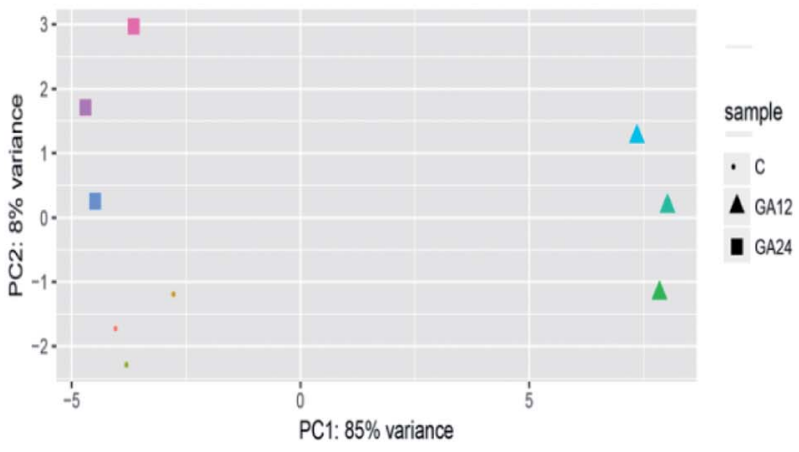

Fig. 3 Gene expression profiles of HCT116 cells treated with GA. (a) Hierarchical clustering of differentially expressed genes in HCT116 cells based on $\log _{10}$-transformed expression values (RPKM). (b) Principle component analysis (PCA) on the complete transcriptome of HCT116 cells in the GA treatment group (GA group) and control group (C group).

Principal component analysis suggested that similar samples within a group clustered together, which was in agreement with the heat map (Fig. 3b). The first two principal coordinates explained total $93 \%$ of the variation observed among all the groups. The cells treated with GA for $12 \mathrm{~h}$ formed a distinct cluster from the untreated cells and cells treated for $24 \mathrm{~h}$.

For differential gene expression analysis, pairwise comparisons of datasets from HCT116 cells treated with GA at different time points were performed. Only genes with a significant fold change (fold change $\geq 2 ; P<0.05$ ) were included in this analysis. As shown in Fig. 4 a, the expressions of 61 genes $(7.7 \%)$ were increased and $730(92.3 \%)$ genes were decreased after $12 \mathrm{~h}$ GA treatment. In contrast, over $93.2 \%$ differentially expressed genes (DEGs) (849 genes) were up-regulated and only 6.8\% DEGs (62 genes) were down-regulated during the treatment progress from $12 \mathrm{~h}$ to $24 \mathrm{~h}$. There were 40 transcripts that fluctuated in the GA24 group compared with the untreated group C. We further identified the shared DEGs between each pairwise comparison groups and the numbers of DEGs among these samples are visualized in the Venn diagrams (Fig. 4b). Transcriptional profiling showed that 648 DEGs were modulated both in the first $12 \mathrm{~h}$ and later $12 \mathrm{~h}$ of the treatment process. Notably, the regulation pattern of these shared 648 genes was discrepant during the first $12 \mathrm{~h}$ and later $12 \mathrm{~h}$ treatment process. 611 down-regulated genes in the GA12-C group were turned into up-regulation in the GA24-GA12 group. 37 up-regulated genes in the GA12-C group then were down-regulated in the GA24-GA12 group. 14 genes DEGs were detected both in GA24-C group and GA24-GA12 group.

\section{GO and KEGG enrichment analysis of DEGs}

To understand the functions of the DEGs and the biological processes involved in GA treatment to HCT116 cells, we carried out GO enrichment analysis and classified them into three independent GO categories. Each DEG could be assigned to more than two GO terms and the top 30 enriched GO terms are shown in Fig. 5a. A total 738 out of 791 DEGs from the C-GA12 group could be assigned to a total of $4185 \mathrm{GO}$ terms, including 3219 biological process terms, 558 molecular function terms and 408 cellular component terms. According to the three main categories of the GO classification, "calcium ion binding", the "plasma membrane", the "extracellular region" and "single-multicellular organism process" were most prevalent. Only a few DEGs classified under "Ras guanyl-nucleotide exchange factor activity" and "developmental maturation" were also observed.

As treatment time extended, the number of DEGs increased. About 92.8\% (845 out of 911) DEGs identified between GA12 and GA24 were categorized into 4577 GO terms. The "calcium
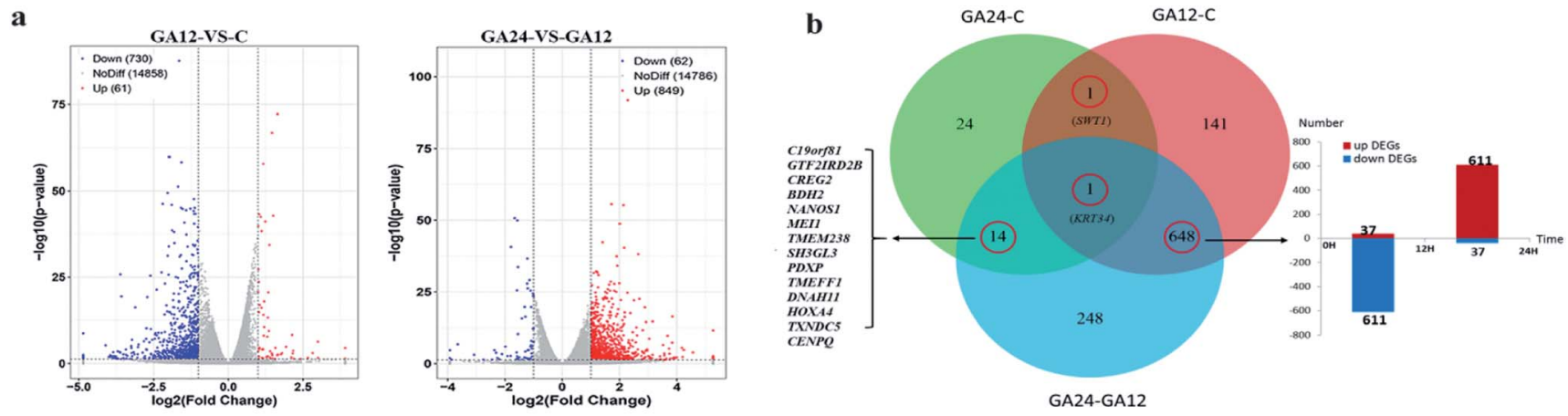

Fig. 4 Overview of the differentially expressed genes for each comparison samples. (a) Volcano plots of differentially expressed genes between treated group GA12 with control group C, and treated group GA24 with treated group GA12 (b) Venn diagram depicting the differentially expressed genes common to different experimental exposure time. 
$\mathbf{a}$
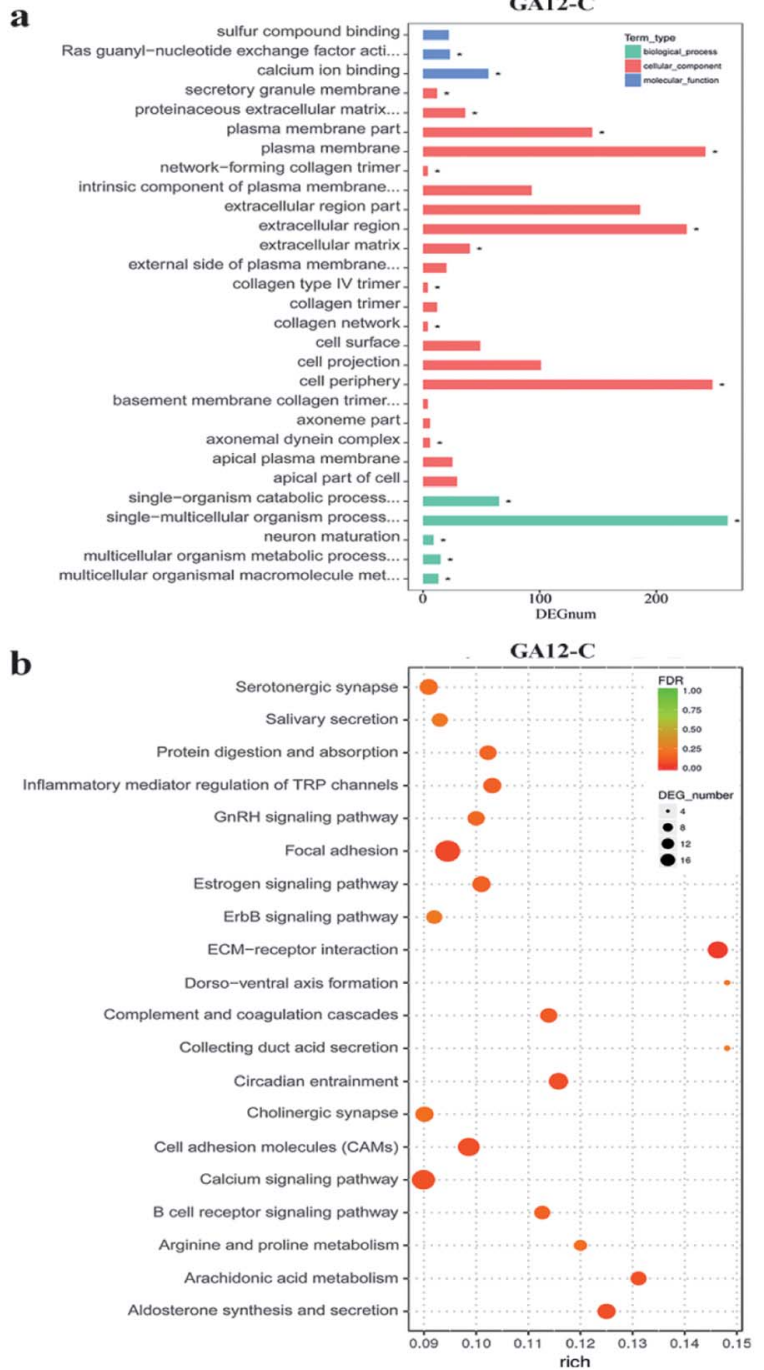

GA12-C

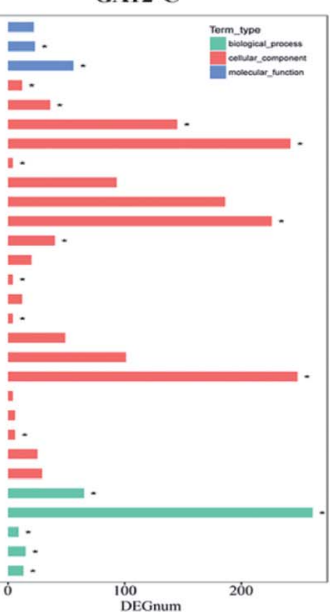

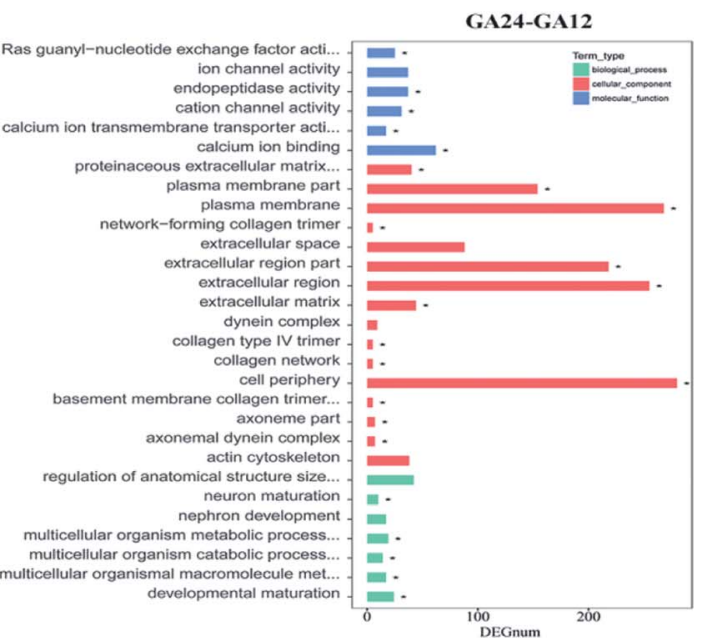

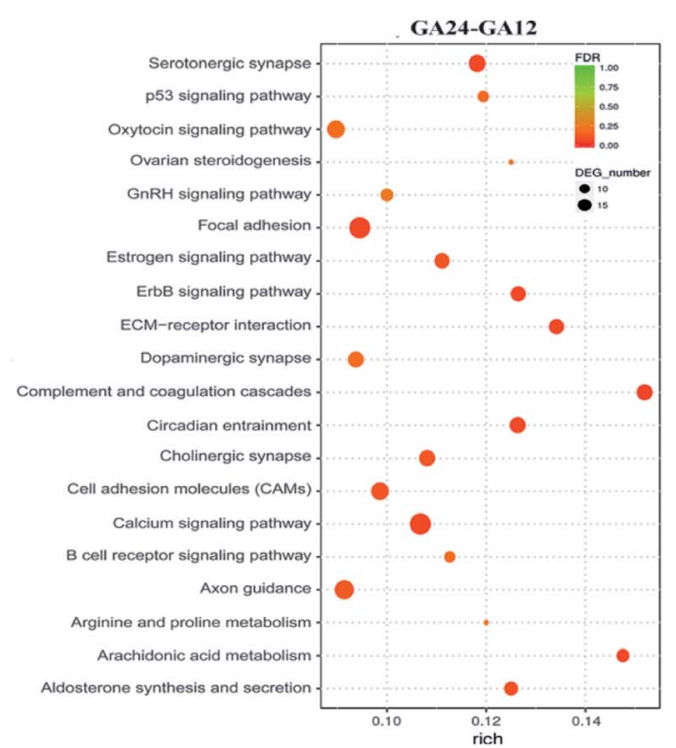

Fig. 5 GO and KEGG enrichment analysis of differentially expressed genes. (a) Histograms of the top 30 enriched GO terms. Enriched items were selected with a corrected. $p$-Value 0.05. (b) Scatter plots of the top 20 enriched KEGG pathway terms. Enriched items were measured by the rich factor, $q$ value $(q<0.05)$ and the number of genes.

ion binding" and related channel, "plasma membrane", "extracellular region" and "cell periphery" showed the highest enrichment and the "network-forming collagen trimer" and "multicellular organism metabolic process" were much lower enrichment GO terms. Only the GO term "FMN binding" was significantly enriched when compared the untreated samples (C group) to cells treated with GA for $24 \mathrm{~h}$ (GA24 group).

All the DEGs were mapped to the reference pathway in the KEGG database to identify the response pathway operating during the treatment process. However, only a few genes could be annotated to the KEGG pathways and most were involved metabolism and signal transduction. Fig. 5b displays the top 20 significantly enriched KEGG pathways for the DEGs during the first $12 \mathrm{~h}$ and the later $12 \mathrm{~h}$ treatment with GA. Of the 791 DEGs detected in GA12 samples, only 178 had a KO ID and were associated with 119 pathways. The enriched pathways were mainly related to "Organismal Systems" and "Environmental Information Processing". Among these pathways, the "calcium signaling pathway, "inflammatory mediator regulation of TRP channels", "cholinergic synapse" and "ErbB signaling pathway" were highly enriched. The enriched "arginine and proline metabolism" and "circadian entrainment", which were related to lipid metabolism and environmental adaptation were also detected. Further exposure to GA induced more DEGs, of which 182 genes have predicted involvement in 192 pathways. The enriched pathways were mainly related to "organismal systems" and "environmental information processing". The highly enriched pathways were "Calcium signalling pathway", "Arachidonic acid metabolism", "Cell adhesion molecules (CAMs)" and "ErbB signalling pathway". Two pathways "focal adhesion" and "p53 signalling pathway" associated with "cellular community" and "cell growth and death" were also observed.

\section{Metabolic pathways related to proliferation and apoptosis}

Genes from the top 10 significantly enriched pathways could be considered as the important gene expression regulators in response to GA treatment, especially the ones shared between the C-GA12 group and GA12-GA24 group (Table 1). Most gene 
Table 1 The significantly enriched KEGG pathways and the identified genes between treated cells and untreated cells ${ }^{a}$

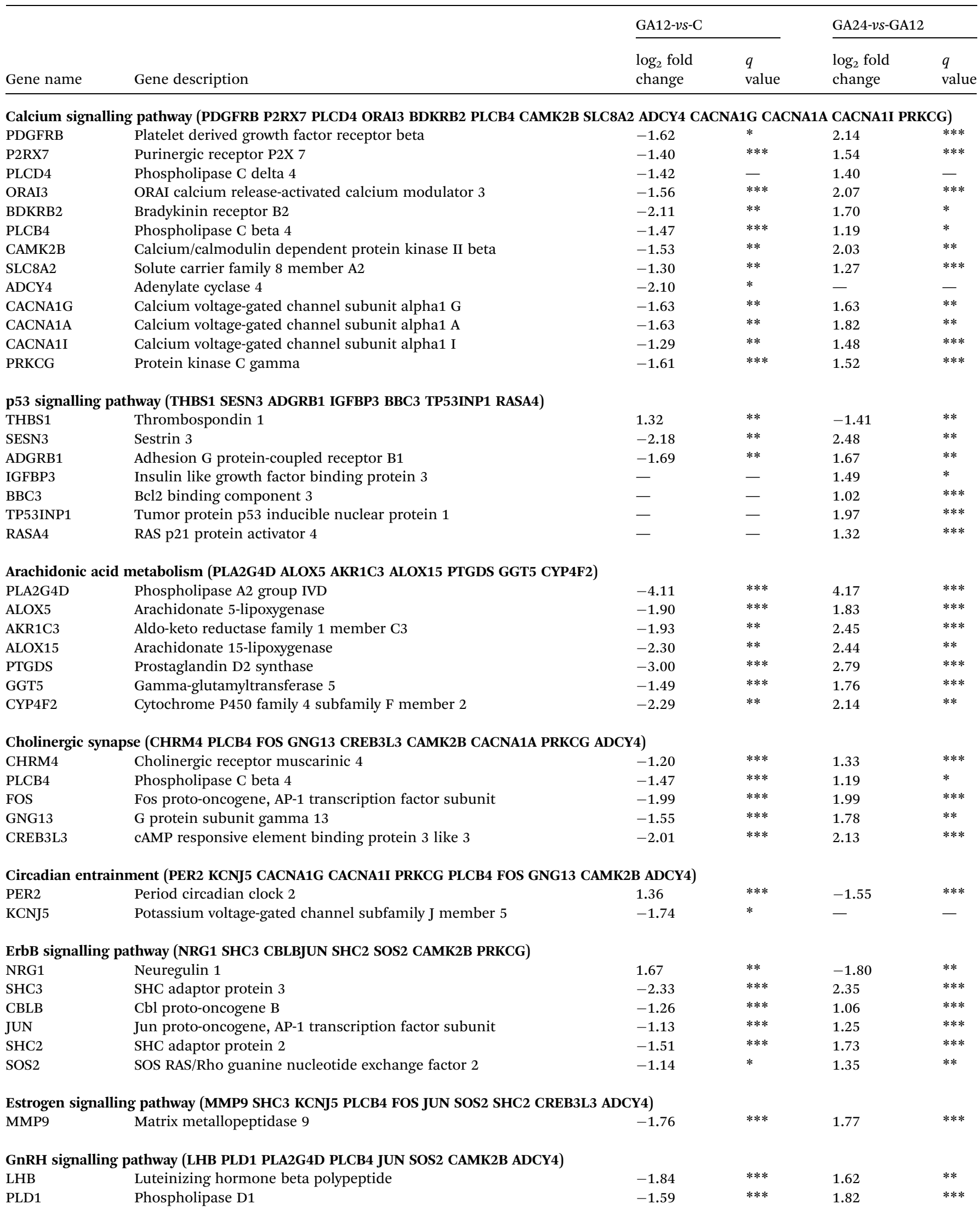




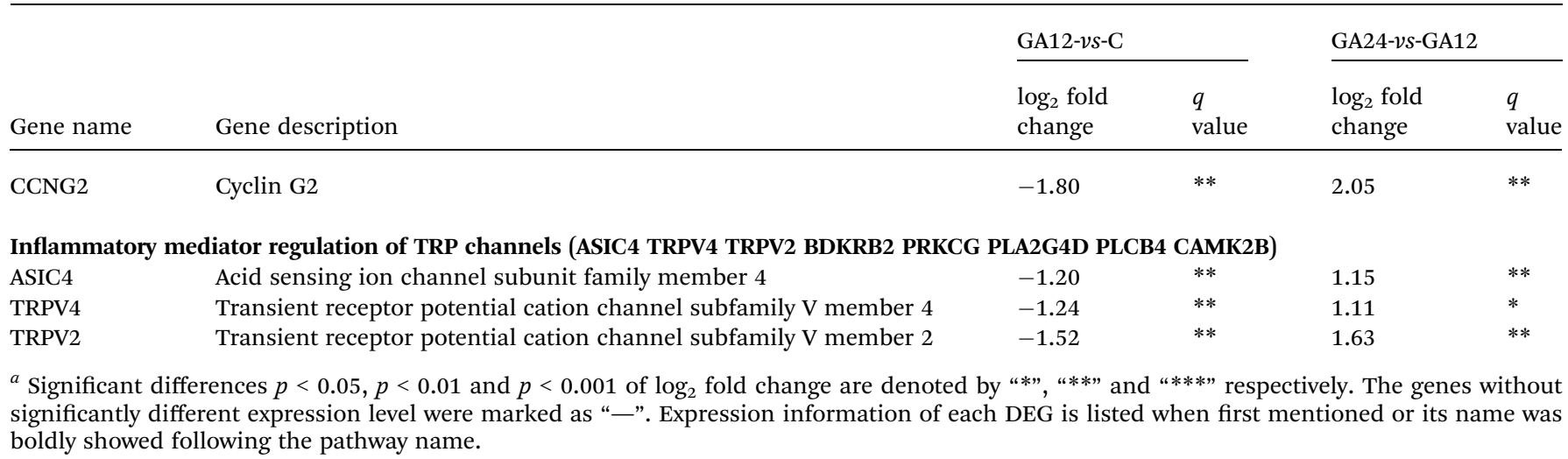

expression patterns among other pathways also showed initial down-regulation and then up-regulation during the $24 \mathrm{~h}$ treatment. But the gene Per2 encoding period circadian clock 2 in the circadian entrainment pathway, gene NRG1 encoding neuregulin 1, and gene THBS1 encoding thrombospondin 1 were up-regulated in the C-GA12 group and down-regulated in the GA12-GA24 group. Genes involved in the arachidonic acid metabolism pathway were down-regulated in the first $12 \mathrm{~h}$ and then up-regulated in the last $12 \mathrm{~h}$, such as ALOX5 and ALOX15 genes encoding members of the lipoxygenase gene family, which catalyze the synthesis of leukotrienes from arachidonic acid. The calcium signalling pathway involved the most number of DEGs, among them, CACNA1G, CACNA1A, CACNA1I encoded calcium voltage-gated channel (VGC) subunits. Purinergic receptor P2RX7 belonged to receptor-operated calcium channel (ROC), and calcium release-activated calcium modulator ORAI3 was considering as store-operated calcium channels (SOC). They were all found down-regulated in GA12-C group and upregulated in GA24-GA12. Two transmembrane protein-coding genes PDGFRB, BDKRB2 encoding signal receptors were also found down-regulated first and then up-regulated. The left four DEGs in the calcium signal pathway: CAMK2B, PLCD4, PLCB4, ADCY4 and PRKCG genes encoding important enzymes also differentially expressed in other 7 pathways including cholinergic synapse, circadian entrainment, ErbB signalling pathway, estrogen signalling pathway, serotonergic synapse GnRH signalling pathway and Inflammatory mediator regulation of TRP channels. There were 7 DEGs found in the p53 signalling pathway, THBS1, SESN3 and ADGRB1 were down-regulated in the first $12 \mathrm{~h}$ and then up-regulated in the last $12 \mathrm{~h}$ as the same as most DEGs. However, TP53INP1, RASA4, IGFBP3 and BBC3 which are directly related to cell cycle and death, were upregulated only in the GA12-GA24 group.

Based on the GO and KEGG enrichment analysis, the primary fluctuation in HCT116 cells induced by GA treatment were sketched. As shown in Fig. 6, after exposure to GA for $12 \mathrm{~h}$, the stress was generated and detected by the sensor-like proteins such as P2RX7, PDGFRB and BDKRB2 on the cell membrane. At the same time, the calcium release-activated calcium modulator (ORAI3) and the voltage-gated calcium channel proteins (CACNA1A, CACNA1I, and CACNA1G) were down-regulated and the concentration of calcium in the cytoplasm was restrained. Changed calcium signal was sensed through intermediate calcium sensors to decrease the activity of nitric oxide synthase (NOS1) and calcium/calmodulin (CALM)dependent protein kinase (CAMK2B). The down-regulation of CAMK2B could lead to the arrest of the cell cycle and inhibit cell proliferation. NOS1 and protein kinase C (PRKCG), which are mediated by the intracellular calcium and second messenger diacylglycerol (DAG), also would affect other pathways involved in cell communication and the phosphatidylinositol signal pathway. This indicates that in order to respond to GA, HCT116 cells might reprogram the gene expression to optimize signaling transduction for more efficient and effective adaptation.

With the increase of exposure time, the down-regulated DEGs in the calcium signal pathway became up-regulated after $24 \mathrm{~h}$ of treatment. The expression level of four genes in the p53 signal pathway showed no significant differences in the GA12-C comparing group but were markedly enhanced in the GA12GA24 group. TP53INP1 is a p53 target gene involved in a large panel of cellular processes, such as reactive oxygen species regulation, apoptosis, and cell cycle. The high expression level of TP53INP1 implicated in a positive feedback loop with transcriptional activity of p53. p53 directly activates the expression of many genes important for cell cycle regulation and apoptosis through binding to p53 DNA-binding sites. The up-regulation of Bcl2-binding component 3 (BBC3, also known as PUMA), a proapoptotic protein, would led to the release of cytochrome $\mathrm{C}$ and induction of cell apoptosis through the mitochondrion pathway. Increased IGFBP3 level would also induce cell apoptosis and suppress the IGF-1/mTOR pathway. Although there was no significant change in the p21 gene, the up-regulated RASA4 who associated with its activation was detected, which suggested that the G1 arrest of the cell cycle was also affected.

\section{Validation RNA-seq by RT-qPCR}

We randomly selected 12 DEGs involved in different metabolism pathways to verify their expression levels. The information of used primers and the calculated fold change in RT-qPCR experiments were shown in Table 2 . The fold change values of each gene are the 


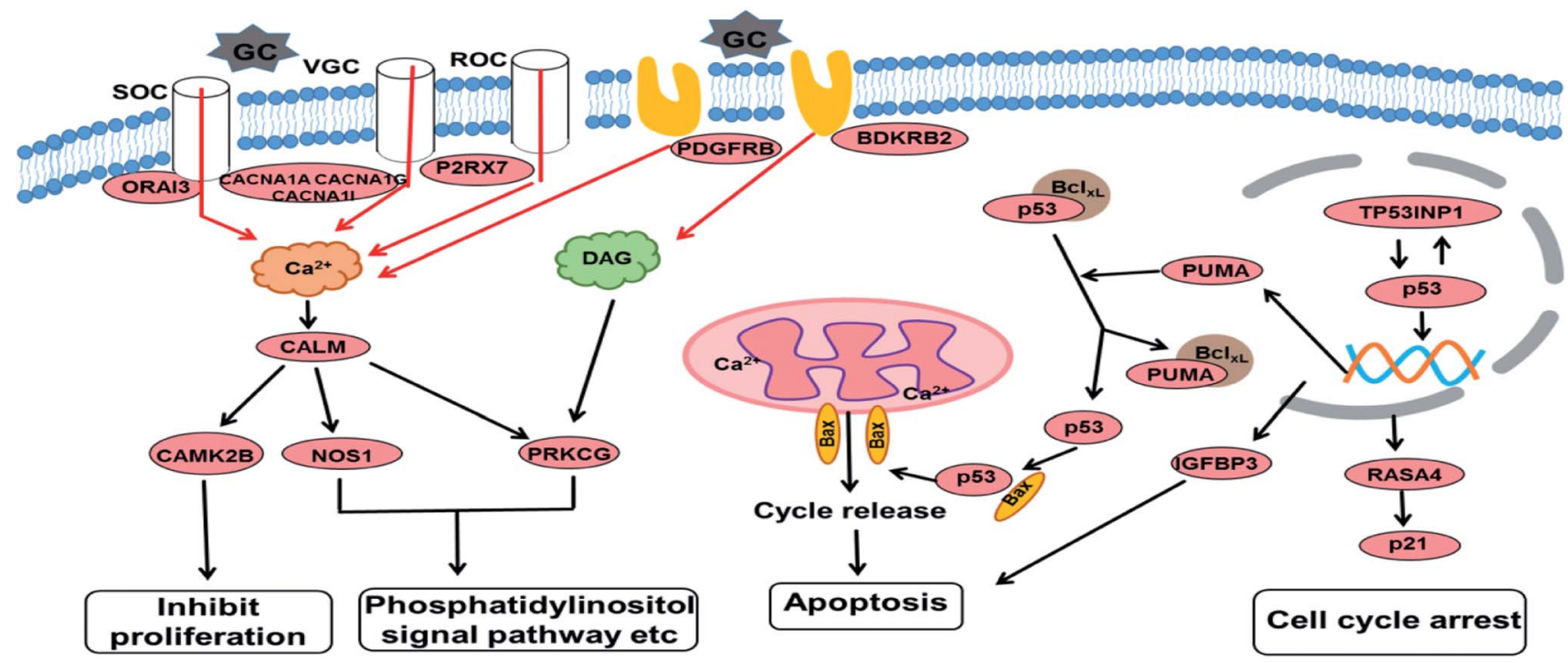

Fig. 6 An overview of the molecular changes and their related functional outcomes induced by GA treatment in HCT116 cells.

Table 2 Primers used in RT-qPCR and the fold change for the selected different expressed genes

\begin{tabular}{|c|c|c|c|c|c|c|c|}
\hline \multirow[b]{2}{*}{ Name } & \multirow[b]{2}{*}{ NCBI_GI } & \multirow[b]{2}{*}{ Forward primer } & \multirow[b]{2}{*}{ Reverse primer } & \multicolumn{2}{|c|}{$\begin{array}{l}\log _{2} \text { fold change } \\
(\mathrm{GA12} / \mathrm{C})\end{array}$} & \multicolumn{2}{|c|}{$\begin{array}{l}\log _{2} \text { fold change } \\
\text { (GA24/GA12) }\end{array}$} \\
\hline & & & & qRT-PCR & RNA-seq & qRT-PCR & RNA-seq \\
\hline ALOX5 & NM_00698.4 & TGGCGCGGTGGATTCATAC & CGTCCATCCCTCAGGACAAC & -1.12 & -1.9 & 1.32 & 1.83 \\
\hline CAMK2B & NM_172084.2 & CGGAATTTCTCAGCCCGGAA & TGAGCCGGATGTAAGCGATG & -0.97 & -1.53 & 1.78 & 2.03 \\
\hline PRKCG & NM_002739.4 & GCCACTAGGTGTCCCCAAC & GAGAATATCGGGCTCCGCTC & -1.03 & -1.61 & 1.12 & 1.52 \\
\hline GNAZ & NM_002073.3 & AGCGGCTACGACCTGAAACT & AAGCAGATGGTGAGCGGGAT & -0.81 & -1.37 & 0.94 & 1.41 \\
\hline FOS & NM_005252.3 & AGGGCTGGCGTTGTGAAGA & TCAGCAGGTTGGCAATCTCG & -1.36 & -1.99 & 1.56 & 1.99 \\
\hline PLCD4 & NM_032726.3 & CAGGGCTTCACCATTGTCTT & TAACCGCTGAACTTCTTGGA & -0.78 & -1.42 & 1.11 & 1.4 \\
\hline CYP4F2 & NM_023944.3 & CCTTGGACAGTCTACAGAAATGC & CTGAGTGGGGAGGGTGCGAC & -1.84 & -2.29 & 1.56 & 2.14 \\
\hline PLAC1 & NM_001316887.1 & TACACGAGGAGTCTGTCAAGG & CAGCCAATCAGATAATGAACCA & -1.03 & -1.66 & 1.09 & 1.66 \\
\hline CCNG2 & NM_004354.2 & CTGAGTTTGATTGAGGCTAC & GTTTCACCTTCATAAGAGCC & -1.22 & -1.8 & 1.17 & 2.05 \\
\hline SESN3 & NM_001271594.1 & TTGACAAGAGGACCAAGTGC & AACTGGCTCCGCAAGAAAGA & -1.34 & -2.18 & 1.36 & 2.48 \\
\hline KRT6A & NM_005554.3 & CATCCAAGAGGTCACCGTCA & GCAGGGTCCACTTTGTTTCC & 2.57 & 3.92 & -4.15 & -3.93 \\
\hline MASP2 & NM_006610.3 & GAGGACATTGACGAGTGCCA & TGGAGAGTTTGGGATACGGC & 1.58 & 2.13 & -2.11 & 1.29 \\
\hline
\end{tabular}

mean values of three parallel experiments. The RT-qPCR results were consistent with the RNA-seq findings, indicating that the RNA-seq results were quite credible.

\section{Discussion}

Gallic acid abundantly exist in several natural food and food products, including spices and drinks. More and more studies revealed an efficacy of GA administration to various health scenarios such as cardiovascular diseases, ${ }^{24}$ hypertension, ${ }^{25}$ hepatic ailments, ${ }^{26}$ or cancers ${ }^{27}$ in vivo experiments. For colorectal cancer, diet is thought to play a major role in its etiology. Gallic acid and gallates from dietary supplementation may exert beneficial effects and there is increasing evidence providing a basis for using them in the prevention and treatment of colorectal cancer. ${ }^{28}$

In this study, we investigated whether GA inhibit proliferation in HCT116 cells and sought to identify the mechanisms of GA related to cell apoptosis. Our findings suggested that GA induced apoptosis of colon cancer cell lines through calcium and p53 signal pathways, which indicate GA as a novel promising therapy for the treatment of colon cancer.

Our results showed that GA could time- and dosedependently inhibit cell proliferation and induce apoptosis as measured by CCK-8 assay and Annexin V-staining assay. We also used RNA-seq technology to determine the global regulation network for the response of HCT116 cells to GA treatment and identified some key pathways related to cell apoptosis.

GA was cytotoxic to HCT116 cells in a time- and dosedependent manner, which was superior to the effect of GA on HCT-15 cell proliferation. ${ }^{29}$ As reported by Henning et al., one cup of green tea and black tea contains approximately $17 \mathrm{mg} \mathrm{GA} .^{30}$ The $\mathrm{IC}_{50}$ of GA on HCT116 cells after $24 \mathrm{~h}$ of treatment was approximately $54.8 \mu \mathrm{M}$ and this concentration could be easily reached in the gastrointestinal tract, as $3 \mathrm{mg}$ of GA is enough to yield about 88 $\mu \mathrm{mol} \mathrm{L}{ }^{-1}$ in the colonic volume of $200 \mathrm{~mL}$. These results show that GA has insignificant anti-proliferative activity against colon cancer cells at a very low concentration within the range of biological availability. 
Flow cytometric analysis revealed that the early and late apoptotic cell proportions increased along with increasing GA concentration. Furthermore, cell viability only exhibited a significant drop between $12 \mathrm{~h}$ and $24 \mathrm{~h}$ treatment by GA at concentration higher than $50 \mu \mathrm{M}$. These results suggested that the inhibition of the proliferation of HCT116 cells by GA relies on inducing the typical apoptotic pathway and massive death of cells probably occurred during 12 to $24 \mathrm{~h}$ exposure to GA.

Transcriptome data can offer accurate genome information quickly and economically, especially in revealing impacts on biological pathways and processes that had previously not been considered. ${ }^{31}$ We identified 792 and 911 genes with known functions that were differentially expressed in $12 \mathrm{~h}$ and $24 \mathrm{~h}$ GA-treated HCT116 cells, respectively. These tremendous changes reflect not only protein cascades directly related to apoptosis, but also many genes and transcription factors involved in calcium channels, circadian entrainment, serotonergic synapse and fatty acid metabolism. The fluctuant DEGs involving cell cycle detected in HCT116 cells indicate that like other anticancer agents, GA has the ability to disturb the cell cycle in cancer cells, which might contribute to the induction of apoptosis. ${ }^{32}$ Our results also revealed that GA modulated the expression of many cell signaling molecules in the p53 signal pathway, such as Bcl2 binding component $\mathrm{BBC} 3$, which is agreement with previous studies showing that GA triggered the intrinsic or mitochondrial pathway to lead to enhanced cancer cell death. ${ }^{33,34}$

The calcium ion $\left(\mathrm{Ca}^{2+}\right)$ is one of the key intracellular signals that is implicated in the regulation of cell functions such as impregnation, cell proliferation, differentiation and death. ${ }^{35}$ Cells use this external source of $\mathrm{Ca}^{2+}$ by activating various entry channels with widely different properties. ${ }^{36}$ In the first $12 \mathrm{~h}$ of treatment with GA, considerable calcium channels subunitcoding genes involved in three calcium channels were downregulated. These calcium channels mediate the entry of calcium ions into excitable cells, and thus their downregulation in the early phase of GA exposure would decrease the intracellular calcium concentration. Moreover, the expression level of two membrane protein-coding genes PDGFR and BDKRB2 were also decreased. Although the functional pathway between these proteins and GA was unclear, suppressed expression of these factors could activate multiple downstream targets inside the cell to decrease intracellular free calcium. Protein CaMK2B which is involved in many signaling cascades is regulated by the $\mathrm{Ca}^{2+} / \mathrm{CALM}$ complex. ${ }^{37}$ We inferred that the down-regulation of this gene was caused by loss of intracellular calcium and finally lead to suppress of the cell proliferation process in the first $12 \mathrm{~h}$ exposure to GA.

The calcium signal also plays an important role in cell death and survival; there is a close relation among intracellular $\mathrm{Ca}^{2+}$ concentration, Bcl 2 family proteins and cell apoptosis. ${ }^{35}$ Recent studies have shown that several pro-apoptotic factors including members of the $\mathrm{Bcl} 2$ family proteins and reactive oxygen species regulate $\mathrm{Ca}^{2+}$ sensitivity through both the $\mathrm{Ca}^{2+}$ release channels in the endoplasmic reticulum and mitochondria. ${ }^{38}$ In this study, with the prolongation of GA treatment time to $24 \mathrm{~h}$, the expression pattern of factors in the calcium signaling pathway was changed to up-regulation. However, the cell proliferation continued to be suppressed and the proportion of cell apoptosis reached as high as $62.2 \%$. A previous report showed that cadmium elevated the intracellular concentration of $\mathrm{Ca}^{2+}$ and CALM and finally induced apoptosis in gill cells of crabs. ${ }^{39}$ Lange et $a .^{40}$ also reported an increased calcium signaling pathway that resulted in apoptosis in neuroblastoma cells. These researches showed that the high concentration intracellular $\mathrm{Ca}^{2+}$ and CALM were closely related to apoptosis in cells enduring chemical stress. Our results indicate that long time GA treatment induced an alteration of calcium signaling pathway genes and increased mitochondria calcium, which eventually led to activation of the apoptotic pathway. ${ }^{41}$

Induction of apoptosis is considered an effective approach to prevent and treat cancer. The tumor suppressor protein p53 plays a crucial role in the regulation of cell cycle arrest and induction of carcinoma cell apoptosis. ${ }^{42}$ p53 exhibits its anticancer role through triggering the expression of genes involved in cell cycle regulation and apoptosis, which ultimately results in the inhibition of cancer progression and malignant transformation. ${ }^{43}$ p53 inhibits cell cycle progression at the G1 or G2/ $\mathrm{M}$ phases through induction of the cell cycle inhibitor p21 and promotes apoptosis through induction of pro-apoptotic Bax expression. ${ }^{44}$ Previous reports showed that the treatment of a polyphenol-curcumin could upregulate total and activated p53, as well as increase the expression of p53-regulated p21, PUMA and Bax in human colon cancer. ${ }^{45}$ It was reviewed that increased $\mathrm{Bcl} 2$ family members including PUMA, NOXA, and BIM, induced the mitochondrial apoptotic pathway by triggering Bak/Bax activation. ${ }^{46}$ In this study, p53 inducible nuclear protein TP53INP1, BBC3 (PUMA) and p21 activated kinase RASA4 were overexpressed after $24 \mathrm{~h}$ treatment of GA. Thus we inferred that the up-regulated p53 signal pathway triggered mitochondrial apoptosis in HCT116 cells.

In summary, here we investigated the effect and mechanism of the phenolic compound GA on the growth of HCT116 colon cancer cells by RNA-seq. KEGG analysis showed that the DEGs mainly encoded proteins involved in cholinergic synapse, circadian entrainment, calcium signal processing and transport, arachidonic acid metabolism and the p53 signal pathway. The regulation of the expression of calcium ion binding proteins and related channels between the first $12 \mathrm{~h}$ and later $12 \mathrm{~h}$ after treatment with GA suggests that the calcium signal pathway plays important roles in both cell proliferation and cell apoptosis. The results also suggest that GA-triggered apoptosis in human colon cancer HCT116 cells was associated with the up-regulation of the intrinsic p53 signal pathway through the activation of caspases and finally led to apoptosis through the mitochondrial pathway. Our findings reveal the mechanism of GA on inducing colon cancer cells and also provide insight for GA as a potential chemotherapeutic agent for colon cancer. However, further investigation in clinical settings are needed to promote GA as a likely candidate for anticancer agent.

\section{Conflicts of interest}

There are no conflicts to declare. 


\section{Acknowledgements}

This work was supported by the Agricultural Science and Technology Innovation Project of Chinese Academy of Agricultural Sciences (CAAS-ASTIP-2013-OCRI), the National Natural Science Foundation of China (31701560) and Director Fund of Oil Crops Research Institute (1610172017009).

\section{References}

1 A. W. Lowe and R. H. Moseley, Gastroenterology, 2012, 143, 1125-1126.

2 A. Jemal, F. Bray, M. M. Center, J. Ferlay, E. Ward and D. Forman, Ca-Cancer J. Clin., 2011, 61, 69-90.

3 H. F. A. Vasen, I. Tomlinson and A. Castells, Nat. Rev. Gastroenterol. Hepatol., 2015, 12, 88-97.

4 L. Anning, N. Koo, J. Neely, A. Wells, S. K. Clark, R. Miller and O. Will, Colorectal Dis., 2011, 13, E297-E302.

5 E. P. Misiakos, N. P. Karidis and G. Kouraklis, World J. Gastroenterol., 2011, 17, 4067-4075.

6 G. D. Stoner and H. Mukhtar, J. Cell. Biochem., 1995, 169180.

7 J. R. Araujo, P. Goncalves and F. Martel, Nutr. Res., 2011, 31, 77-87.

8 J. Liu, H. T. Wu, J. F. Lu, X. Y. Wen, J. Kan and C. H. Jin, Chem. Eng. J., 2015, 262, 803-812.

9 J. Serrano, R. Puupponen-Pimia, A. Dauer, A. M. Aura and F. Saura-Calixto, Mol. Nutr. Food Res., 2009, 53, S310-S329.

10 D. Li, Z. J. Liu, W. J. Zhao, Y. L. Xi and F. L. Niu, Process Biochem., 2011, 46, 2210-2214.

11 A. Abdelwahed, I. Bouhlel, I. Skandrani, K. Valenti, M. Kadri, P. Guiraud, R. Steiman, A. M. Mariotte, K. Ghedira, F. Laporte, M. G. Dijoux-Franca and L. Chekir-Ghedira, Chem.-Biol. Interact., 2007, 165, 1-13.

12 S. H. Kim, C. D. Jun, K. Suk, B. J. Choi, H. Lim, S. Park, S. H. Lee, H. Y. Shin, D. K. Kim and T. Y. Shin, Toxicol. Sci., 2006, 91, 123-131.

13 M. S. Kang, J. S. Oh, I. C. Kang, S. J. Hong and C. H. Choi, J. Microbiol., 2008, 46, 744-750.

14 J. M. Kratz, C. R. Andrighetti-Frohner, P. C. Leal, R. J. Nunes, R. A. Yunes, E. Trybala, T. Bergstrom, C. R. Barardi and C. M. Simoes, Biol. Pharm. Bull., 2008, 31, 903-907.

15 M. Inoue, R. Suzuki, T. Koide, N. Sakaguchi, Y. Ogihara and Y. Yabu, Biochem. Biophys. Res. Commun., 1994, 204, 898-904.

16 M. Kawada, Y. Ohno, Y. Ri, T. Ikoma, H. Yuugetu, T. Asai, M. Watanabe, N. Yasuda, S. Akao, G. Takemura, S. Minatoguchi, K. Gotoh, H. Fujiwara and K. Fukuda, Anticancer Drugs, 2001, 12, 847-852.

17 K. Wang, X. Zhu, K. Zhang, L. Zhu and F. F. Zhou, J. Biochem. Mol. Toxicol., 2014, 28, 387-393.

18 S. W. Weng, S. C. Hsu, H. C. Liu, B. C. Ji, J. C. Lien, F. S. Yu, K. C. Liu, K. C. Lai, J. P. Lin and J. G. Chung, Anticancer Res., 2015, 35, 2077-2084.

19 M. Inoue, R. Suzuki, N. Sakaguchi, Z. Li, T. Takeda, Y. Ogihara, B. Y. Jiang and Y. Chen, Biol. Pharm. Bull., 1995, 18, 1526-1530.

20 A. Mortazavi, B. A. Williams, K. Mccue, L. Schaeffer and B. Wold, Nat. Methods, 2008, 5, 621-628.
21 K. W. J. Derks, B. Misovic, M. C. G. N. van den Hout, C. E. M. Kockx, C. P. Gomez, R. W. W. Brouwer, H. Vrieling, J. H. J. Hoeijmakers, W. F. J. van IJcken and J. Pothof, $R N A$ Biol., 2015, 12, 30-42.

22 U. Nagalakshmi, K. Waern and M. Snyder, Curr Protoc Mol Biol., 2010, ch. 4, Unit 4, vol. 11, pp. 11-13.

23 K. O. Mutz, A. Heilkenbrinker, M. Lonne, J. G. Walter and F. Stahl, Curr. Opin. Biotechnol., 2013, 24, 22-30.

24 J. R. Xie and L. N. Yu, Acta Anaesthesiol. Scand., 2007, 51, 909913.

25 L. Jin, Z. H. Piao, S. M. Sun, B. Liu, G. R. Kim, Y. M. Seok, M. Q. Lin, Y. Ryu, S. Y. Choi, H. J. Kee and M. H. Jeong, Sci. Rep., 2017, 7, 15607.

26 O. Karimi-Khouzani, E. Heidarian and S. A. Amini, Pharmacol. Rep., 2017, 69, 830-835.

27 H. A. Aglan, H. H. Ahmed, S. A. El-Toumy and N. S. Mahmoud, Tumor Biol., 2017, 39, 6.

28 R. Y. Kosuru, A. Roy, S. K. Das and S. Bera, Mol. Nutr. Food Res., 2018, 62, 1700699.

29 A. P. Subramanian, S. K. Jaganathan, M. Mandal, E. Supriyanto and I. I. Muhamad, World J Gastroenterol., 2016, 22, 3952-3961.

30 S. M. Henning, P. Wang, N. Abgaryan, R. Vicinanza, D. M. de Oliveira, Y. Zhang, R. P. Lee, C. L. Carpenter, W. J. Aronson and D. Heber, Mol. Nutr. Food Res., 2013, 57, 483-493.

31 S. R. Zhao, W. P. Fung-Leung, A. Bittner, K. Ngo and X. J. Liu, PLoS One, 2014, 9, e7864.

32 M. D. Garrett and I. Collins, Trends Pharmacol. Sci., 2011, 32, 308-316.

33 R. X. Wang, L. J. Ma, D. Weng, J. H. Yao, X. Y. Liu and F. G. Jin, Oncol. Rep., 2016, 35, 3075-3083.

34 K. Wang, X. Zhu, K. Zhang, L. Zhu and F. Zhou, J. Biochem. Mol. Toxicol., 2014, 28, 387-393.

35 A. Danese, S. Patergnani, M. Bonora, M. R. Wieckowski, M. Previati, C. Giorgi and P. Pinton, Biochim. Biophys. Acta, 2017, 1858, 615-627.

36 S. W. Jones, J. Bioenerg. Biomembr., 2003, 35, 461-475.

37 T. Yamauchi, Biol. Pharm. Bull., 2005, 28, 1342-1354.

38 M. Swami, Nat. Rev. Cancer, 2010, 10, 738.

39 J. X. Wang, P. P. Zhang, N. Liu, Q. Wang, J. X. Luo and L. Wang, PLoS One, 2015, 10, e0144392.

40 I. Lange, J. Moschny, K. Tamanyan, M. Khutsishvili, D. Atha, R. P. Borris and D. L. Koomoa, Int. J. Oncol., 2016, 48, 16081616.

41 X. Ma, X. Tian, X. Huang, F. Yan and D. Qiao, Mol. Cell. Biochem., 2007, 302, 99-109.

42 X. D. Ma, X. M. Tian, X. X. Huang, F. Yan and D. F. Qiao, Mol. Cell. Biochem., 2007, 302, 99-109.

43 S. Kim and S. S. A. An, Medicine, 2016, 95, e3993.

44 F. B. Charrier-Savournin, M. T. Chateau, V. Gire, J. Sedivy, J. Piette and V. Dulic, Mol. Biol. Cell, 2004, 15, 3965-3976.

45 J. L. Watson, R. Hill, P. B. Yaffe, A. Greenshields, M. Walsh, P. W. Lee, C. A. Giacomantonio and D. W. Hoskin, Cancer Lett., 2010, 297, 1-8.

46 R. J. Youle and A. Strasser, Nat. Rev. Mol. Cell Biol., 2008, 9, 47-59. 